\title{
Research on Millimeter Wave Fuze Echo Signal-to-Noise Ratio under \\ Projectile Motion
}

\author{
Jie Zhou ${ }^{1, a^{*}}$, Shuo $\mathrm{Wu}^{2, \mathrm{~b}}$, Haibin $\mathrm{Wu}^{3, \mathrm{c}}$ \\ ${ }^{1,2,3}$ Army Officer Academy,Hefei,Anhui,230031,China \\ a962854399@qq.com,b3025508944@qq.com, ${ }^{\mathrm{c} 3456245312 @ q q . c o m}$
}

\begin{abstract}
Keywords:millimeter wave fuze; SNR; projectile motion model; target scattering characteristics Abstract: In order to calculate the millimeter wave proximity fuze echo signal to noise ratio accurately,analyzes the factors that influence the motion of the projectile fuze echo signal to noise ratio of fuze and target distance, fuse and target grazing angle and target to the scattering characteristics and other factors with the projectile motion of fuze echo signal to noise ratio influence. Finally, make the simulation analysis. The simulation results show that the projectile motion of fuze echo signal-to-noise ratio influence must be considered in design of fuze optimal signal processor.
\end{abstract}

\section{Introduction}

Active millimeter wave fuze using radar system, through the transmitting antenna send active millimeter wave signal to the target, receiving antenna receive target's echo signal, which is processed by the signal processor and generate fuze initiation instructions. Thus, echo signal quality directly influence fuze detonating performance in analysis and evaluation of fuze echo signal quality and signal to noise ratio is a key index. Relevant data show that ${ }^{[1]}$, the echo signal to noise ratio mainly depends on the distance of fuze and target, the fuze launch signal and target of the grazing angle and target to scattering characteristics and other factors. Projectile toward its target, these factors change in time, when the study of fuze echo signal to noise ratio, we must study of projectile motion when the factors of fuze echo signal to noise.

\section{Fuze echo SNR analysis with projectile motion}

\section{The fuze antenna configuration and beam pointing}

In order to increase the fuze target detection distance, usually the fuze antenna configuration on the head of the body, emission antenna beam center and projectile axis consistent, specific configuration effects such as shown in Fig 1, which, $\alpha$ is fuze antenna beam center and ground grazing angle, $\beta$ is fuze beam width, $\theta$ is fuze incident angles.

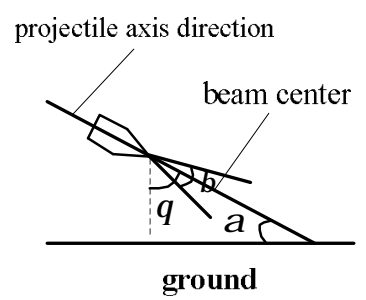

Fig.1. Schematic diagram of the fuze antenna configuration and beam pointing 


\section{Analyze fuze grazing angle with the movement of projectile}

In order to facilitate the research, the rules in Fig 2 (a), to the major axis of the ellipse mn connection with Ox shaft vertical projection of 0 degrees,fuze with the projectile motion process, fuze antenna beam projection change. Axis of projectile angle $\alpha$ with the ground, due to antenna beam center and the body axis coincidence, therefore, fuze antenna beam center and ground grazing angle for $\alpha$.

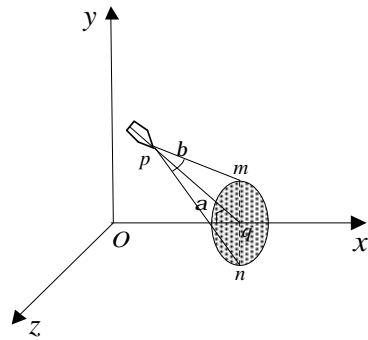

(a)projection 0 degrees

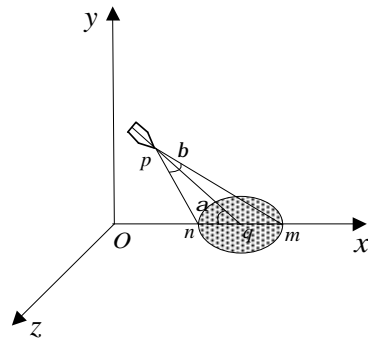

(b)projection 90 degrees

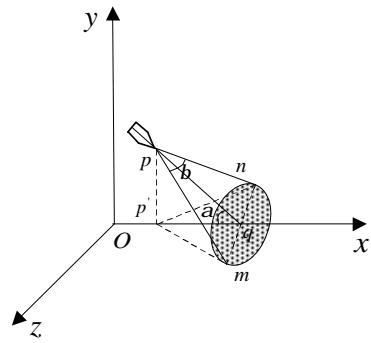

(c)arbitrary angle projection

Fig.2. Schematic diagram of the fuze beam projection

The fuze antenna beam and ground projection can be divided into the following three conditions:

$\square$ The projection of 0 degrees,composed of fuze antenna beam plane pmn and Ox axis angle is $\alpha$,as shown in Fig 2(a).

$\square$ The projection of 90 degrees, $\angle O n p=\alpha+\frac{\beta}{2}, \angle O m p=\alpha-\frac{\beta}{2}$, the maximum grazing angle is $\alpha+\frac{\beta}{2}$, as shown in Fig $2(b)$.

$\square$ Arbitrary projection angle, the point $\mathrm{p}$ to $\mathrm{xoz}$ plane projection, projection point $p^{\prime}$,the largest grazing angle is $\alpha$, as shown in Fig 2(c).

\section{Backscattering coefficient with grazing angle variation analysis of Fuze}

This part of scholars home and abroad to establish a target model of backscattering characteristics. In contrast, the calculation Ulaby model has higher precision than the Kulemin model.

(1)Ulaby terrain scattering coefficients model

F.T.Ulaby and M.C.Dobson analyzed a lot of ground about the scattering test data,put forward the average backward scattering coefficient of empirical model ${ }^{[5]}$ :

$$
\sigma^{0}=P_{1}+P_{2} \exp \left(-P_{3} \theta\right)+P_{4} \cos \left(P_{5} \theta+P_{6}\right)
$$

In the formula, $\theta$ is the fuze incident angle ( $\mathrm{rad}), P_{1} \sim P_{6}$ are parameters, can be obtained by fitting the experimental data.

(2)Kulemin terrain scattering coefficients model

The proposed Kulemin model is suitable for frequency in the range of $3 \sim 100 \mathrm{GHz}$, the empirical model is as follows ${ }^{[5]}$ :

$$
\sigma^{0}=A_{1}+A_{2} \log (\psi / 20)+A_{3} \log (f / 10)
$$


In the formula, $f$ is fuze frequency $(\mathrm{GHz}), \psi$ is grazing angle(degree), $A_{1} \sim A_{3}$ are parameters, With different goals and different ground, in which the coefficient of cement floor, height less than $0.5 \mathrm{~m}$ grassland as shown in table 1 .

Table 1 model parameters Kulemin experience

\section{Echo SNR calculation}

\begin{tabular}{|c|c|c|c|}
\hline Target type & $A_{1}$ & $A_{2}$ & $A_{3}$ \\
\hline Cement floor & -49 & 32 & 20 \\
\hline $\begin{array}{c}\text { The grass height is } \\
\text { less than } 0.5 \mathrm{~m}\end{array}$ & -21 & 10 & 6 \\
\hline
\end{tabular}

The fuze use pulse Doppler radar system, the pulse radar equation is the following form ${ }^{[2]}$ :

$$
\begin{aligned}
& R_{\max }=\left[\frac{P_{a v} G^{2} \lambda^{2} \omega_{P D}}{(4 \pi)^{3} P_{\text {min } \min }(S / N) L^{\prime}}\right]^{1 / 4} \\
& (\mathrm{~S} / \mathrm{N})=\frac{\mathrm{P}_{\mathrm{aV}} \mathrm{G}^{2} \lambda^{2} \sigma \mathrm{D}_{\mathrm{PD}}}{(4 \pi)^{3} \mathrm{P}_{\text {min,min }} \mathrm{R}^{4} \mathrm{~L}^{\prime}}
\end{aligned}
$$

According to the target RCS analysis :

$$
\sigma=\sigma^{0} \mathrm{dA} \approx \frac{\sigma^{0} \mathrm{R} \beta \mathrm{r}_{\mathrm{s}}}{\sin \theta}
$$

Combined formula (4) and (5):

$$
(S / N)=\frac{P_{a v} G^{2} \lambda^{2} D_{P D}}{(4 \pi)^{3} P_{\min , \min } R^{3} L^{\prime}} \frac{\sigma^{0} \beta r_{s}}{\sin \theta}
$$

In formula, $P_{a v}$ is the average sending power of fuze, $G$ is fuze antenna gain, $\lambda$ is fuze wavelength, $\mathrm{R}$ is distance to target, $\sigma^{0}$ is the radar scattering coefficient, $\beta$ is fuze beam width, $r_{s}$ is the fuze of the radial distance resolution, $\theta$ is fuze incident angles, $L^{\prime}$ is sum of Fuze loss and equipment allowance.

$$
D_{P D}=\frac{T_{s}^{2}}{T T_{g}}
$$

Among them, $T_{g}$ is the distance gate, $T_{s}$ is effective pulse width for the echo. The sensitivity of the receiver $P_{\min , \min }=K T_{0} B_{N} F, \mathrm{~K}$ is Pohl Seidman constant, $T_{0}$ is the system temperature, $B_{N}$ is the equivalent noise bandwidth, $\mathrm{F}$ is noise coefficient. 


\section{Simulation analysis}

\section{Projectile motion simulation}

The simulation parameter settings: projectile velocity $950 \mathrm{~m} / \mathrm{s}$, emission angle of 15 degrees, the initial distance of 0 in $\mathrm{X}$ axis and the initial distance of 0 in $\mathrm{Y}$ axis. The angle between the axis of projectile fuze and the ground, the fuze and the ground radial distance is simulated,the simulation results are shown in Fig 3.

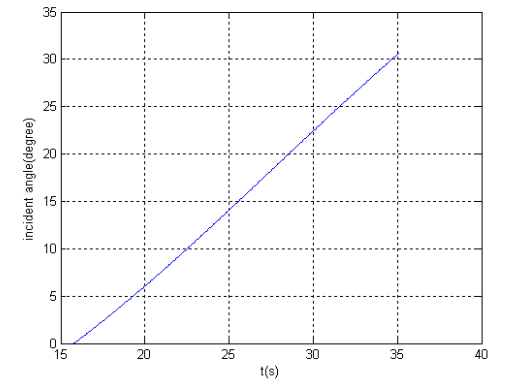

(a)The descending axis of projectile and the ground angle

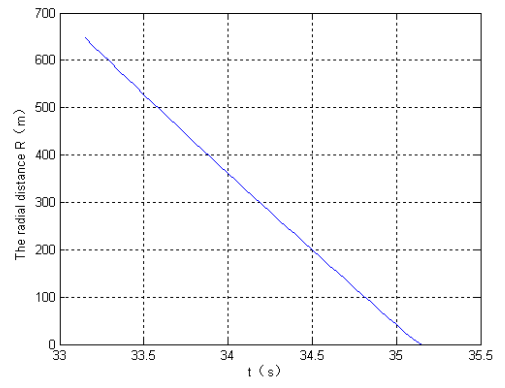

(b) The fuze and radial distance from the ground

Fig.3. the projectile motion simulation map

\section{Grazing angle simulation of Fuze}

The simulation parameter settings: fuze beam width $\beta=20^{\circ}$,fuze simulation with the grazing angle of projectile motion are shown in Fig 4.

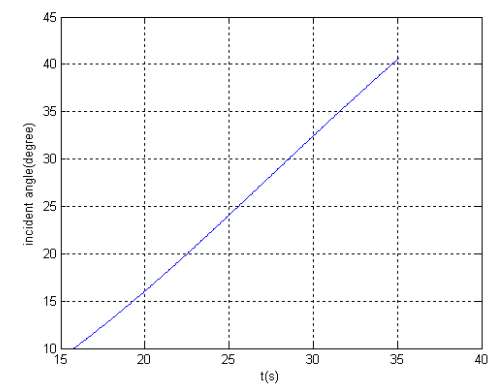

Fig 4 grazing angle fuze simulation map

\section{Fuze echo SNR simulation}

Set the main simulation parameters: $P_{a v}=50 \mathrm{~mW}, G=14 \mathrm{~dB}, \lambda=5 \mathrm{~mm}, \quad \beta=20^{\circ}, r_{s}=3 \mathrm{~m}$, $L^{\prime}=8 d B, T^{0}=300 K, K=1.3806488 \times 10^{-23} J / K, F=6 d B, B_{N}=50 \mathrm{MHz} \circ$ Simulation results is shown in Fig 5. 


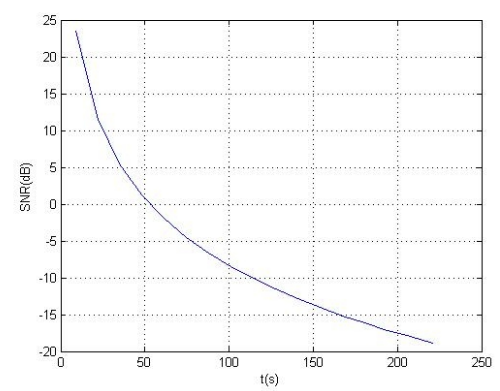

(a)The cement ground Ulaby model simulation map

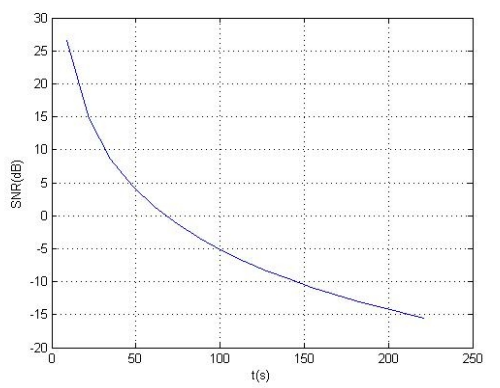

(b)The grass Kulemin model simulation map

Fig.5. simulation of fuze echo signal-to-noise

\section{Conclusion}

The emission of the distance for the fuze and target, the fuze target signal and the grazing angle and the target to the scattering characteristics and other factors of fuze echo signal to noise ratio were theoretical research, and a simulation analysis was carried out. Simulation results show that in the study of fuze echo signal to noise ratio, not only to consider the influence of the above factors, but also consider the impact of different features of fuze echo signal to noise ratio.

\section{References}

[1] Zhao Huichang. Radio fuze design principle and method [M]. Beijing: National Defense Industry Press, 2012.03

[2] Cui Zhanzhong, Song Shihe, Xu Lixin. The principle of proximity fuze (Third Edition( [M]. Beijing: Beijing Institute of Technology press, 2009.08

[3] Song Piji. The exterior ballistics of guns and rockets [M]. Beijing: Weapon Industry Press, 1993.09

[4] Liu Yixin, Liu Yuwen. The exterior ballistics [M]. Beijing: tide press, 1998.12

[5] Zhou Weiyi. Millimeter wave radar clutter characteristics analysis and research of [D]. Nanjing University of Science and Technology master thesis, 2008.06 